Cyanine dyes with tail length asymmetry enhance photoselection: A multiscale study on DiD probes in a liquid disordered membrane Peer-reviewed author version

Paloncyova, Marketa; Aniander, Gustav; Larsson, Emma \& KNIPPENBERG, Stefan (2020) Cyanine dyes with tail length asymmetry enhance photoselection: A multiscale study on DiD probes in a liquid disordered membrane. In:

SPECTROCHIMICA ACTA PART A-MOLECULAR AND BIOMOLECULAR SPECTROSCOPY, 224 (Art No 117329).

DOI: 10.1016/j.saa.2019.117329

Handle: http://hdl.handle.net/1942/29978 


\title{
Cyanine dyes with tail length asymmetry enhance photoselection: a multiscale study on DiD probes in a liquid disordered membrane
}

\author{
Markéta Paloncýová ${ }^{1,2}$, Gustav Aniander $^{1}$, Emma Larsson $^{1}$, Stefan Knippenberg ${ }^{1,2,3,4^{*}}$ \\ ${ }^{1}$ Department of Theoretical Chemistry and Biology, KTH Royal Institute of Technology, \\ Stockholm, Sweden \\ ${ }^{2}$ Biomedical Research Institute, Hasselt University, Agoralaan Building C, 3590 Diepenbeek, Belgium \\ ${ }^{3}$ RCPTM, Department of Physical Chemistry, Fac. Sciences, Palacký University, Olomouc, Czech \\ Republic \\ ${ }^{4}$ Research group PLASMANT, Department of Chemistry, University of Antwerp, Universiteitsplein 1, \\ 2610 Wilrijk-Antwerp, Belgium
}

Author to whom correspondence should be sent: stefan.knippenberg@ upol.cz

\begin{abstract}
Visualization of membrane domains like lipid rafts in natural or artificial membranes is a crucial task for cell biology. For this purpose, fluorescence microscopy is often used. Since fluorescing probes in lipid membranes partition specifically in e.g. local liquid disordered or liquid ordered environments, the consequent changes in their orientation and location are both theoretically and experimentally of interest. Here we focused on a liquid disordered membrane phase and performed molecular dynamics (MD) simulations of the indocarbocyanine $\mathrm{DiD}$ probes by varying the length of the attached alkyl tails and also the length of the cyanine backbone. From the probed compounds in a DOPC lipid bilayer at ambient temperature, a varying orientation of the transition dipole moment was observed, which is crucial for fluorescence microscopy and which, through photoselection, was found to be surprisingly more effective for asymmetric probes than for the symmetric ones. Furthermore, we observed that the orientation of the probes was dependent on the tail length; with the methyls or propyls attached, DiD oriented with its tails facing the water, contrary to the ones with longer tails. With advanced hybrid QM/MM calculations we show that the different local environment for differently oriented probes affected the one-photon absorption spectra, that was blue-shifted for the short-tailed DiD with respect to the DiDs with longer tails. We show here that the presented probes can be successfully used for fluorescence microscopy and we believe that the described properties bring further insight for the experimental use of these probes.
\end{abstract}

\section{Keywords}

Lipid bilayer, DiD, cyanine probe, fluorescence spectroscopy, absorption, hybrid quantum mechanics - molecular mechanics

\section{Introduction}

Studying lipids is one of the most crucial tasks in cell biology. Lipids are one of the constituents of cells membranes that separate the interior environment of the cells from outside and regulate the flux of biomolecules and xenobiotics into the cells [1]. The function of cell membranes is, however, not only 
as a physical barrier. A significant proportion of enzymes in our body interact with membranes or even become a part of the membrane, such as pores, transporters, drug metabolizing enzymes etc. [2-4] and their function is significantly affected by the composition of the membrane [5], in which the lipids are the most important constituents arranged into a lipid bilayer.

The lipid composition of the membranes governs the adopted phase [5]. In artificial, single component membranes, lipids with long saturated tails tend to adopt a gel phase at body temperature, short or unsaturated one adopt the fluid phase. Addition of cholesterol into lipid membranes facilitates formation of the liquid-ordered phase. Natural cell membranes are composed of a huge variety of lipids and these lipid mixtures tend to form domains of specific phase, thickness, permeability etc. [6,7] The ordered domains accommodate a variety of membrane proteins and their visualization is then a crucial task for cell biology.

For visualization of lipid membranes fluorescence techniques are often used. Especially effective is fluorescence microscopy, in particular when lasers are used which bear a high signal to noise ratio $[8,9]$. For this technique, fluorescence probes are embedded into the membrane. The laser beam scans the surface of the membrane and excites the fluorophores. The orientation of the probe is crucial due to the photoselection. For the underlying theory the reader is referred to fluorescence textbooks, such as Lakowicz [10]. In short, the resulting effect is that the efficiency of the excitation is governed by the angle $\theta$ between the electric field vector of the polarized excitation light and the transition dipole moment (TDM) of the probe following a $\cos ^{2} \theta$ dependence. In the case of oriented lipid bilayers and the excitation beam perpendicular to the membrane plane, this practically means that the most efficient probes for fluorescence microscopy lay with their TDM parallel to the membrane plane.

A probe often used in fluorescence microscopy is the cyanine derivative 1,1'-dioctadecyl-3,3,3', $3^{\prime}$ tetramethylindodicarbo-cyanine (DiD, Figure 1) [9,11]. Until recently, DiD has most commonly been known to reside in liquid disordered membrane phases which consist mostly of unsaturated lipids with rather limited tail lengths. However, DiD derivatives with longer tails are found in liquid ordered phases for which DiD's fluorescence emission can be used as a visualization tool $[12,13]$. In our previous work, we proved that DiD can be found in liquid ordered membranes, too, using a well defined ratio of sphingolipids, phospholipids and cholesterol [14]. In all these works, DiD orients itself roughly parallel to the membrane surface [15] and as such is a well working probe for optical techniques in biological tissues. The two long saturated tails (octadecyl) attached to the cyanine backbone are incorporated within lipid tails $[14,16]$. Rather than highlighting the differences of the DiD derivatives for the various lipid phases, we focus in the current study on the influence of the tails on the trajectories and inherent properties of the probes in the liquid disordered phase. Following a study of Ackerman et al., the indocarbocyanine probes are found to disturb their environment only locally and have a negligible effect on the lipid patches of a few hundred lipids [17]. Gullapalli et al. discussed the location and orientation of a probe with a cyanine bridge of only three atoms through molecular dynamics (MD) simulations in a high temperature DPPC lipid bilayer [18]. In line with other theory oriented studies discussing the role of a hydrocarbon tail in anchoring and tuning the location of fluorescent probes in membranes [1923], it can now be assessed if the short-chain hydrocarbon substituents play a minor role in the positioning of the dye, whilst systematically probing to assess the influence of the longer tail.

The electronic structure of the cyanine backbone of the DiD dye is rather interesting and knowledge of its position and orientation with respect to its environment is known to be decisive for the linear and non-linear optical properties. For the cyanines, the relatively large gap between the one-photon allowed first excited state and two-photon allowed second excited state is of importance for its properties and has been the subject of various methodological studies [24-28]. Depending on the position of e.g. counter-ions with respect to the cyanine, its geometry gets symmetry broken and the mean bond length alternations are enhanced. As a consequence, also the two-photon absorption cross sections for the lowest excited states are increased [29]. The use of these (non) linear optical techniques depends on the 
area of interest: in all-optical switching applications and telecommunication, it is considered a loss which should be avoided while in microscopy, it can be used to visualize or discriminate between areas with different characteristics [30]. In the case of $\mathrm{DiD}$, we proved that the SHG data obtained by means of hyper-Rayleigh scattering and by electric field induced second harmonic generation reveal differences in polarization of the probe in different membranes and membrane phases. In a liquid ordered, 2:1 SM:Chol membrane mixture, it has been seen that the DiD 18-18 probe was positioned very high in the membrane and experienced profound influence of the water layer [14]. This particular location was reflected by the two-photon absorption results, which differed from the ones obtained for simulations of the probe in other membranes, where the photochromic part of the dye was located in between the lipid tails [16].

A natural question which arises further is whether the spectroscopic insights can be maximized by manipulating the tails, which is relatively easy to achieve through the synthesis process. To what extent can the tails of the probe affect the rules of photoselection, which are found to be dependent on the orientation of the probe with respect to an external laboratory frame? If it can be assumed that the external electric field of light is applied with a field vector parallel to the membrane plane, it is known that the angle of the transition dipole of the fluorophore should be almost parallel to the field vector. This principle becomes even more restrictive in case of multi photon absorption [10].

Another point whereby the effect can be exploited through the development of cyanine based membrane probes focusses on the changing electronic properties of the DiD-like probes when the backbone is extended. The ratio of the energy of the first and second excited state stays constant when the length increases [28,31], while through the principle of a particle in a box, the excitation energies as a whole decrease. Consequently, the fluorescence energies decrease, too: the member of the DiD family with a cyanine backbone with only 3 carbon atoms fluoresces in orange, while the ones with 5 and 7 carbon atoms are seen in the red and infrared, respectively [32,33]. Following the excitation energies, the chances for photodamage through the use of these probes decrease, too.

The choice for a probe is therefore a delicate one as advantages and disadvantages have to be weighted out against each other. In the current study, we focused on the consequences of slightly modified geometries of the DiD probe upon its behaviour in the lipid bilayer membranes. We modified the length of its tails from one to 22 carbons, varied the saturation of its tails as well as the length of the conjugated backbone (from five to nine carbons, see Figure 1 and Table 1). We analysed their location, orientation and surrounding environment in order to evaluate their performance as probes for the fluorescence microscopy.<smiles>[R2]n1c(C=CC=CC=CC2(C)C(=CC=CC)C(C)(C)c3ccccc32)[n+]([R7])c2ccccc21</smiles>

Figure 1: Structure of DiDs, $R_{1}, R_{2}$ and $n$ for individual molecules are listed in Table 1.

Table 1: Cyanine backbone or alkyl tails`lengths $\left(n, R_{1}, R_{2}\right.$; see Figure 1) and the efficiency of the photoselection in fluorescence microscopy $\left\langle\cos ^{2} \theta>(\theta\right.$ is the angle of TDM in respect of the membrane plane)

\begin{tabular}{|c|c|c|c|c|c|}
\hline & & $n$ & $R_{1}$ & $R_{2}$ & $\left.<\cos ^{2} \theta\right\rangle^{a}$ \\
\hline $1-1$ & \pm & 2 & $\mathrm{CH}_{3}$ & $\mathrm{CH}_{3}$ & 0.72 \\
\hline 3-3 & 离 & 2 & $\left(\mathrm{CH}_{2}\right)_{2} \mathrm{CH}_{3}$ & $\left(\mathrm{CH}_{2}\right)_{2} \mathrm{CH}_{3}$ & 0.86 \\
\hline $6-3$ & $\sum_{4}^{2}$ & 2 & $\left(\mathrm{CH}_{2}\right)_{5} \mathrm{CH}_{3}$ & $\left(\mathrm{CH}_{2}\right)_{2} \mathrm{CH}_{3}$ & 0.92 \\
\hline
\end{tabular}




\begin{tabular}{|c|c|c|c|c|c|}
\hline $10-3$ & & 2 & $\left(\mathrm{CH}_{2}\right)_{9} \mathrm{CH}_{3}$ & $\left(\mathrm{CH}_{2}\right)_{2} \mathrm{CH}_{3}$ & 0.90 \\
\hline $14-3$ & & 2 & $\left(\mathrm{CH}_{2}\right)_{13} \mathrm{CH}_{3}$ & $\left(\mathrm{CH}_{2}\right)_{2} \mathrm{CH}_{3}$ & 0.88 \\
\hline $18-3$ & & 2 & $\left(\mathrm{CH}_{2}\right)_{17} \mathrm{CH}_{3}$ & $\left(\mathrm{CH}_{2}\right)_{2} \mathrm{CH}_{3}$ & 0.83 \\
\hline $22-3$ & & 2 & $\left(\mathrm{CH}_{2}\right)_{21} \mathrm{CH}_{3}$ & $\left(\mathrm{CH}_{2}\right)_{2} \mathrm{CH}_{3}$ & 0.92 \\
\hline $18-12$ & & 2 & $\left(\mathrm{CH}_{2}\right)_{17} \mathrm{CH}_{3}$ & $\left(\mathrm{CH}_{2}\right)_{11} \mathrm{CH}_{3}$ & 0.83 \\
\hline $18-18$ & & 2 & $\left(\mathrm{CH}_{2}\right)_{17} \mathrm{CH}_{3}$ & $\left(\mathrm{CH}_{2}\right)_{17} \mathrm{CH}_{3}$ & 0.81 \\
\hline $18: 0-18: 1$ & 퐆 & 2 & $\left(\mathrm{CH}_{2}\right)_{17} \mathrm{CH}_{3}$ & $\left(\mathrm{CH}_{2}\right)_{8} \mathrm{CH}=\mathrm{CH}\left(\mathrm{CH}_{2}\right)_{7} \mathrm{CH}_{3}$ & 0.74 \\
\hline $18: 1-18: 1$ & 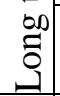 & 2 & $\begin{array}{l}\left(\mathrm{CH}_{2}\right)_{8} \mathrm{CH} \\
\left(\mathrm{CH}_{2}\right)_{7} \mathrm{CH}_{3}\end{array}$ & $\left(\mathrm{CH}_{2}\right)_{8} \mathrm{CH}=\mathrm{CH}\left(\mathrm{CH}_{2}\right)_{7} \mathrm{CH}_{3}$ & 0.81 \\
\hline $\operatorname{DiD}(\mathrm{C} 7)^{b}$ & & 3 & $\left(\mathrm{CH}_{2}\right)_{17} \mathrm{CH}_{3}$ & $\left(\mathrm{CH}_{2}\right)_{17} \mathrm{CH}_{3}$ & 0.77 \\
\hline $\operatorname{DiD}(\mathrm{C} 9)^{b}$ & & 4 & $\left(\mathrm{CH}_{2}\right)_{17} \mathrm{CH}_{3}$ & $\left(\mathrm{CH}_{2}\right)_{17} \mathrm{CH}_{3}$ & 0.87 \\
\hline
\end{tabular}

${ }^{a}<\cos ^{2} \theta>$ is averaged over simulation time

${ }^{\mathrm{b}} \operatorname{DiD}(\mathrm{C} 7)$ and $\mathrm{DiD}(\mathrm{C} 9)$ denote the DiD probes with a cyanine backbone of 7 and 9 carbon atoms, respectively (see value of $n$ ).

\section{Methods}

We studied the DiD probes varying their tail length. These cyanine based probes have a single positive charge in their singlet ground state. Further, we created DiDs with unsaturated tail(s), and we varied the length of the cyanine backbone of DiD from 5 carbons till 7 and 9 carbons. The list of all studied DiD molecules can be found in Figure 1 and Table 1. DiD topologies were built based on a RESP procedure [34] with Gaussian 16 [35] based on an electrostatic potential surface calculated by B3LYP/cc-pVDZ level of theory [36,37] following the so-called Duan [38] model with an IEFPCM diethylether solvent $\left(\varepsilon_{\mathrm{r}}=4.24\right)$. The RESP procedure was performed by Antechamber from the AMBER 11 software package [39]. The geometry parameters were generated by tleap from the same software package, following the same procedure as in ref. [40]. Special attention has been paid to the parametrization of the cyanine backbone dihedral angles, whose potential energy surfaces have been calculated at the quantum chemical CAM-B3LYP/cc-pVDZ level of theory. For atom types we used GROMOS43a1-s3 force field [41], which mimics drug-membrane interactions well [40].

For accelerating the incorporation of DiDs into the membrane and to obtain generally faster dynamics, we used a fluid phase, pre-equilibrated dioleoylphosphatidylcholine (DOPC) bilayer membrane analogous to the ones used in our previous work [42] with 64 lipids per leaflet. Lipids were modelled by a GROMOS 43a1-s3 force field [41,43-45], fully hydrated with the SPC/E model [46]. A physiological $0.15 \mathrm{M}$ concentration of $\mathrm{Na}^{+}$and $\mathrm{Cl}^{-}$ions was maintained. The DiD molecules were added to the water phase at the top of the simulation box; one DiD molecule was inserted per simulation box.

All MD simulations were performed in GROMACS 5.1.4 [47] with a Nosé-Hoover $[48,49]$ thermostat maintaining the temperature to $298 \mathrm{~K}$ (time constant $0.5 \mathrm{ps}$ ) and with an anisotropic Parrinello-Rahman barostat [50] set to 1 bar (time constant $5 \mathrm{ps}$ ). The electrostatic interactions were treated explicitly to $1.4 \mathrm{~nm}$ and a particle-mesh Ewald summation [51] was applied. van der Waals interactions were cut off to $1.4 \mathrm{~nm}$, while LINCS constraints [52] were applied on all bonds. The integration step of $2 \mathrm{fs}$ was used and the simulations were carried out for 400-700 ns. We considered the simulations as converged within $\sim 30 \mathrm{~ns}$ after DiD entered the membrane (center of mass of DiD nitrogens was found below the level of DOPC phosphates) and started the analysis at this time (Table S1). MD simulations have been further analyzed using internal GROMACS tools with the membrane oriented in xy plane with an axis perpendicular to the membrane surface in the $\mathrm{z}$-direction. For the purpose of the position analysis, 0 in the $\mathrm{z}$-axis was placed in the center of mass of the membrane.

For several probes (DiD 1-1, 14-3, 22-3) we performed additional simulations in order to gain more statistical certainty. However, as we did not observe any significant difference in location or orientation (Figures S11 and S12) we focused further on a single run for each of the systems that we present here. 
For the description of the effect of the environment on the optical properties of the probes we calculated one-photon absorption (OPA) spectra for DiD 1-1 and DiD 6-3 in vacuum and membrane environment. For OPA in vacuum we used first excited singlet state energies on the CAM-B3LYP level of theory [53] with the cc-pVDZ basis set [37], same setup was used for OPA in SCRF solvents, diethylether and ethanol. CAM-B3LYP/cc-pVDZ was benchmarked against other TDDFT functionals as well as postHartree Fock methods in previous works by our group and found to correctly reproduce the energetics [54]. For the role of membrane environment we extracted 40 random frames from the last $200 \mathrm{~ns}$ of the MD simulations of DiD 1-1 and DiD 6-3. We applied the same approach as in other works of our group as well as other authors $[14,30,54,55]$. We extracted by a cylindrical cutoff of $2 \mathrm{~nm}$ the lipids around the DiD and a we also used a semispherical cutoff of $1.5 \mathrm{~nm}$ for the solvent. The QM/MM calculations were performed with the electrostatic embedding method implemented in Dalton2016 [56,57]. The system is then described by two different approaches: the DiD probe is described by CAM-B3LYP/ccpVDZ, while the surrounding environment is described by a molecular mechanics force field, which was used in the previous MD simulations. We investigated the absorption spectrum of all 40 frames; the simulated excitation energies gave rise to vertical absorption lines while a Gaussian broadening with a FWHM of $8 \mathrm{~nm}$ has been used to construct the envelope.

\section{Results and Discussion}

\section{DiD parameterization}

The length of the alkyl chain did not influence the charge distribution in the DiD head. We calculated the partial charges by the RESP approach and observed a conserved charge distribution along the cyanine backbone and in most regions of the cyanine rings (Figure S1). The only significant difference (more than $0.1 \mathrm{e}^{-}$) was observed for DiD 1-1, where the nitrogen atoms are slightly positively charged, as well as the methyl groups attached to them. In all other cases the nitrogen atoms were found to have a small negative charge that did not differ significantly between individual molecules.

Further we analysed the energy barriers for the cyanine backbone twisting by potential energy scans along three dihedral angles (the other three are considered as symmetrical images). The potential energy scans revealed significant energy barriers which increased towards the center of the cyanine bridge: it amounts to $15 \mathrm{kcal} / \mathrm{mol}$ for the 'nitro' dihedral angle which starts at the nitrogen atom, reaches 21 $\mathrm{kcal} / \mathrm{mol}$ for the next dihedral angle and gets even to $24 \mathrm{kcal} / \mathrm{mol}$ for the 'mid' one in the middle of the cyanine bridge (Figure S2). Consequently, the rotation was found highly unlikely and a cis-trans isomerization has not been seen in the MD calculations. Since especially these dihedral angles are crucial for a further description of the dye, we fitted the obtained scans with the Ryckaert-Bellemans [58] potential and used the parameters for our MD simulations (Table S2).

\section{MD simulations}

During our simulations, all DiD probes entered the membrane and accommodated below the head groups region (Table S1, Figure S3). We monitored the position of DiDs nitrogen atoms' center of mass (effectively the middle of the cyanine backbone) and observed that the probes with two long tails (bearing 18 carbons, further called 'long tail') is located $\sim 1.5 \mathrm{~nm}$ from the membrane center (Figure 2), in agreement with our previous work [14]. When unsaturations are available in the tails, no significant change in the position was observed. A difference is seen between the DiD probes with a cyanine bridge of 5 carbons and the ones with 7 or 9 carbon atoms, that are located $0.3 \mathrm{~nm}$ and $0.4 \mathrm{~nm}$ deeper, respectively. The longer cyanine backbone behaves here as a larger hydrophobic part of the molecule (with respect to a cyanine bridge of 5 carbons), dragging it deeper into the hydrophobic membrane region.

Most of the DiD derivatives are located deeper in the membrane than the long tail ones. Most of the probes were located around $1.2 \mathrm{~nm}$ from the middle of the membrane (Figure 2). This is valid for asymmetric probes with both a propyl tail and with a tail whose length ranges from six to 22 carbons 
(further called 'asymmetric'). Shortening both tails to propyl (DiD 3-3) or methyl (DiD 1-1) leads again to a further position of the probes (up to $1.6 \mathrm{~nm}$, these probes are further called 'short tail'). All of the groups of probes entered the membrane freely and we did not observe any DiD permeation, in agreement with a penetration barrier of $6 \mathrm{kcal} / \mathrm{mol}$ found through z-constraint simulations for DiD 1818 [14].

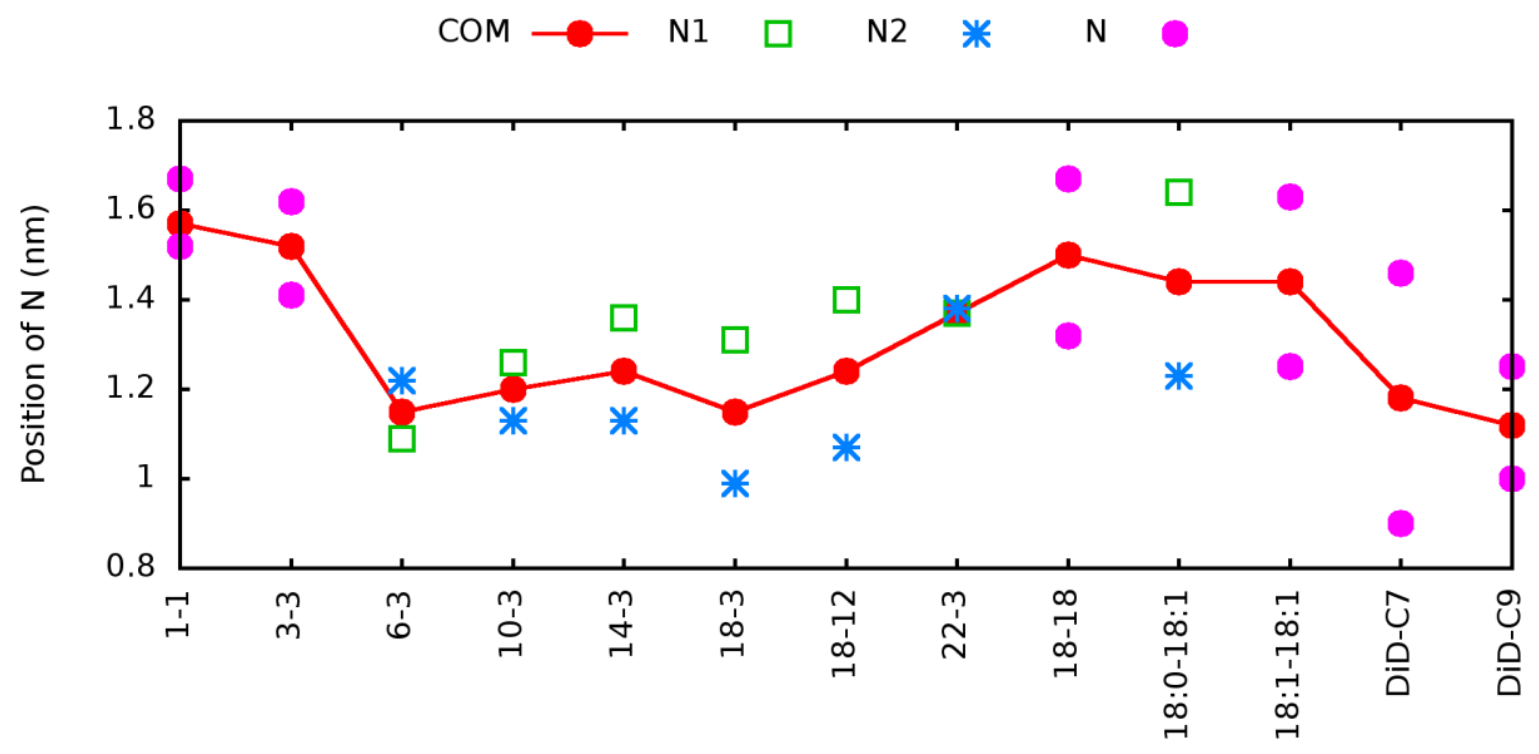

Figure 2: Average position of DiDs' nitrogens COM and the positions of individual nitrogens along the $z$-axis with respect to the middle of the membrane. The nitrogens COM (effectively the middle of the cyanine backbone) is depicted in red; in case of asymmetric probes, $N 1$ (green square) carries shorter alkyl tail, $N 2$ (blue stars) carries longer alkyl tail; in case of symmetric ones the position of nitrogen atoms is shown in magenta points $(N)$. The standard error of the mean is smaller than the symbols used in the plot.

Up till now, it has been generally assumed that the DiDs are oriented with their cyanine backbones parallel to the membrane plane. The orientation of the transition dipole moment (TDM) is important for fluorescence microscopy, and in DiD the TDM is oriented along the cyanine backbone [14]. In the current work, we assume that the electric field vector of a linearly polarized beam, as used in fluorescence microscopy, lays parallel to the membrane plane. Due to the principle of photoselection, the ensemble average of the angle $\theta$ between TDM and the electric field vector governs the efficiency of the excitation essentially through a $\left\langle\cos ^{2} \theta>\right.$ dependence. The highest efficiency was found with 'short tail' probes with average of $\left\langle\cos ^{2} \theta\right\rangle$ of 0.89 (for values for individual probes see Table 1 or S1). Surprisingly, the photoselection efficiency for both symmetric 'long tail' and 'short tail' probes was lower at 0.79 . On the other hand, according to our simulations, the photoselection with a symmetrical DiD molecule with a cyanine bridge of 9 carbons $[\mathrm{DiD}(\mathrm{C} 9)]$ is most opportune $\left(\left\langle\cos ^{2} \theta>=0.87\right)\right.$, corresponding with the most populated $\theta$ at $90^{\circ}$ (Figure S4). We remark that this probe is positioned the deepest in the membrane and is therefore influenced the least by charges present at the top and by water at the interface.

Most populated were the states where the DiD was oriented slightly tilted with the nitrogen bearing a shorter alkyl tail further from the membrane center (Figure 2) and the angle of TDM to the z-axis varied within $\sim 60-105 \mathrm{deg}$ (Table S1). Here the level of tilting does not correlate with the length of the longest tail as e.g. for DiD 22-3 where the cyanine bridge is most probably oriented parallel to the membrane surface. For DiD 18-3, on the other hand, the main angle of TDM to the z-axis already diminished to $75^{\circ}$. These examples express the importance of the length of both tails in the orientation of the probe. For the symmetric $\operatorname{DiD}(\mathrm{C} 5)$ probes, the most probable orientation was around $\sim 65^{\circ}$, as 
well as for $\mathrm{DiD}(\mathrm{C} 7)$. However, the orientation of the TDM was very variable during all simulations (Figure S4).

The length of the tails indeed affects the orientation of the DiD as a whole. In all cases the DiDs were in all-trans conformation (data not shown) and both their alkyl tails faced approximately the same direction. When at least one of the alkyl tails was six or more carbons long, the whole DiD oriented its nitrogen atoms (and correspondingly the alkyl tails) towards the hydrophobic membrane interior (Figures 3, S5). But if both tails were short (a chain of only three carbons or even one single methyl group; 'short tail' probes), the hydrophobic interactions were not strong enough and the nitrogens were facing the membrane/water interface. This explains as well the relatively high position of the center of mass between both nitrogen atoms for these two DiD probes (see Figure 2).

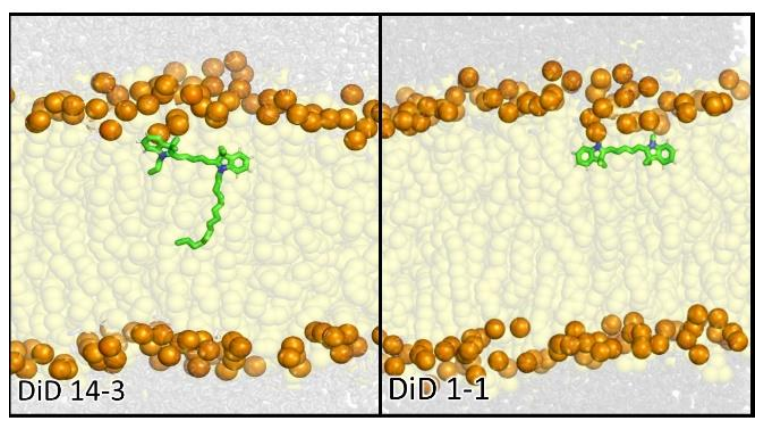

Figure 3: Orientation of DiD with long tails (left) and short tails (right). The DiDs are shown as sticks with green carbons, white hydrogens and blue nitrogens. Lipids are shown as yellow balls with orange phosphate atoms and water molecules are depicted as grey balls.

The longer alkyl tails of the DiDs are organized similarly as lipid tails. The deuterium order parameters $\left|\mathrm{S}_{\mathrm{CD}}\right|$ of DOPC and those of the 'long tail' probes are comparable (Figure S6). As the DiD chains are mostly parallel to lipid tails (Figure $\mathrm{S} 5$ ), $\left|\mathrm{S}_{\mathrm{CD}}\right|$ show that the $\mathrm{DiD}$ chains are slightly less ordered than those of DOPC in that specific depth and this is valid also for unsaturated tails or for the $\mathrm{DiD}(\mathrm{C} 7)$ or $\mathrm{DiD}(\mathrm{C} 9)$. Long tails permeating to the other leaflet are very disordered. In the 'asymmetric' probes, the order parameter for the shorter chains decays steeply to zero and might point therefore at a very disordered behaviour. It can be remarked that we are limited in the current model with a united atom force field and that we only consider here three atoms in a chain, while the $\left|S_{\mathrm{CD}}\right|$ calculation takes the neighbouring carbons into account (see e.g. ref [59] for details). Interesting is the case of DiD 10-3. From the data shown in Figure S5, it is seen that from all probes considered, both tails of DiD 10-3 are the most perpendicularly oriented with respect to the z-axis. An analysis of the order parameters indicates that the order increases along the longer tail of $\mathrm{DiD} 10-3$, but decreases for all other probes.

The longer alkyl tails are embedded in the membrane hydrophobic core. The shorter tails do not reach the membrane middle, from the ten carbons per tail they start to participate in the overall density of the membrane middle (Figure S7, Table S1). Prolonging the alkyl tail even more, to 14 carbons, it is slightly able to intercalate into the opposite membrane leaflet. The level of participation in the density of the original membrane leaflet is dependent on the length of the alkyl tail: whether none, one or both tails reach the middle of the membrane (Figure S7). A deeper position of the cyanine head in $\operatorname{DiD}(C 7)$ and $\mathrm{DiD}(\mathrm{C} 9)$ leads to a higher fraction of $\mathrm{DiD}$ mass in the membrane middle and in the opposite membrane leaflet (Table S1).

Overall, the position and orientation of DiDs are heavily dependent on the length of their alkyl tails. The 'short tail' DiDs ( $<=3$ carbons) orient with the nitrogens and tails facing the water, in all other cases the tails are embedded in between lipid tails. Increasing the length of the tails, the DiDs are 'pulled' into the membrane by hydrophobic interactions. And the 'long tail' ones (18 carbons and more) 
the DiDs are again 'pushed' out of the membrane (Figure 2) due to geometrical constraints - the membrane leaflet is not thick enough to accommodate the whole alkyl tail. As most of the molecules stays in one membrane leaflet and the membrane middle is known for its low density [60], the excess groups of the tails are likely to accommodate mostly in the membrane's centre. Based on the various interactions in a lipid bilayer, it can therefore be said that the longer tails not only adjust themselves with respect to the position of the probe as reported previously [22], but that it can be argued that they also influence it considerably.

Being positioned below lipid head groups, DiDs are in close contact with polar lipid atoms. For 'short tail' DiDs, the cyanine bridge is found at the same distance from the membrane centre as the carbonyl and glycerol oxygens of both DOPC tails (See Figure S8), which is in line with what has been seen in previous studies on NBD phospholipid probes [20,22]. For the 'asymmetric' probes, which are located closer to the membrane's centre, the tilt of the backbone becomes important as the stabilizing electrostatic interactions with polar head groups are only possible for the 'higher' lying part of the probe. Comparing Figure 2 with Figure S8, the orientation of the 'long tail' probes enables a stabilized position of one of the two nitrogen atoms above the lower oxygens of the glycerol groups in the DOPC lipids, while the other nitrogen is found considerably deeper, at a distance in which interactions with lipid oxygens are hardly possible. The double bond in the tails of the DOPC lipids is too deeply buried in the membrane to interact profoundly with the probes. A membrane is furthermore characterized by a balance between repulsive pressure interactions between lipids and a more cohesive effect of hydrophobic tensions which gathers the lipid tails and separates them from the water phase. The position and orientation of the various probes with different tails is therefore an interplay of packing effects, of through space interactions between the cyanine backbone and the headgroups of the lipids, and of steric hindrances.

Not only do the lipid atoms in the surrounding environment differ, but also the hydration of DiDs varies with their position and orientation. The radial distribution function (RDF) of water oxygens around the DiD nitrogen atoms revealed that the water around DiD 1-1 and 3-3 was significantly closer than for the other molecules with the first peak at $\sim 0.5 \mathrm{~nm}$ (Figure S9). The DiD with longer tails have the first peak at $\sim 0.7 \mathrm{~nm}$. Such a different level of hydration can affect the spectroscopical properties of DiDs in the membranes.

\section{One-photon absorption (OPA)}

In order to describe the role of the DiD position and orientation, we calculated OPA spectra for DiD 11 and 6-3 (Figure 4). In vacuum, the major HOMO->LUMO transitions for both probes have similar energies and are therefore seen at $485 \mathrm{~nm}$ for DiD 1-1 and at $488 \mathrm{~nm}$ for DiD 6-3. In diethyl-ether and ethanol, with relative permittivities of 4.24 and 24.85, respectively, the absorption lines are found at $570 \mathrm{~nm}$ and $600 \mathrm{~nm}$ for DiD 1-1 and $570 \mathrm{~nm}$ and $598 \mathrm{~nm}$ for DiD 6-3, respectively. A red shift is thus seen for both molecules when the dielectic constant of the solvent increases. The calculated spectra should be taken with respect to the experimental absorption peak of DiD 1-1, $647 \mathrm{~nm}$ in ethanol [61]. We are aware of the observed difference and focus here rather on the relative differences.

In membranes, these two probes are located at different depths with respect to the water layer and most importantly, they are oriented in different ways. DiD 1-1 is mainly characterized by its tails pointing towards the water layer while DiD 6-3 is oriented with its tails going into the membrane, comparable to all other probes with longer tails (Figure 3). The calculated OPA spectra in the membrane show a red shift in respect to vacuum: the major HOMO - > LUMO peak is now located at $505 \mathrm{~nm}$ for DiD 1-1 
and at $548 \mathrm{~nm}$ for DiD 6-3. The latter is close to the major absorption peak of DiD 18-18, which was simulated previously and for which a wavelength of $558 \mathrm{~nm}$ was found in DOPC [16]. The orientations of DiD 18-18 and DiD 6-3 in the membrane are further on very comparable and these two compounds are found at the same distance from the water layer (Figure S8). It is interesting to note that the red shift of DiD 1-1 in the lipid bilayer is rather limited compared to the one of DiD 6-3, which is located almost $4 \AA$ deeper in the membrane. For DiD 1-1, the nitrogens are in close contact with the polar groups, which can be found all around the probe. DiD 6-3, however, can be found below the charged headgroups of the lipids, which causes a stronger anisotropic polarization of the molecule. The lipid bilayer around this cyanine based probe disturbs thus the symmetric electronic configuration of the dye, which is seen in the expressed bathochromic shift. It can be concluded that the location and orientation of the probes in the membrane significantly influences the resulting spectra. These results are in line with simulations of electric field induced second harmonic generation and hyper Rayleigh scattering for DiD 18-18, which revealed differences in polarization dependent on the probe's surrounding lipid and lipid phase [16].

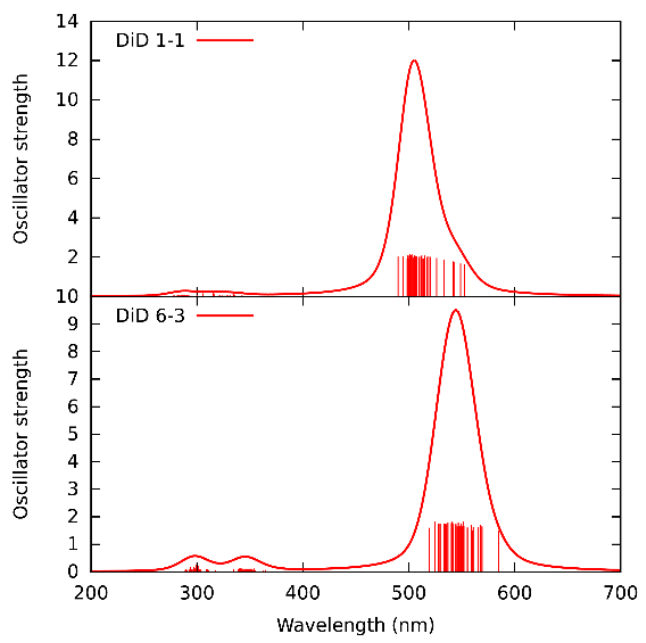

Figure 4: One-photon absorption spectra of DiD 1-1 and DiD 6-3 in DOPC membrane.

\section{Conclusion}

In our study we investigated the DiD derivates with a variation of the alkyl tail lengths. Varying the tail lengths, the major aspects of the $\mathrm{DiD}$ behaviour are still preserved - the probes enter the membranes easily and spontaneously and orient approximately parallel to the membrane surface, generally well for the fluorescence microscopy. For fluorescence microscopy with an incoming field having a transition state dipole moment parallel to the membrane surface, the photoselection for fluorescence microscopy was the most efficient for asymmetric probes like DiD with tails of 6 and 3 carbon atoms. It was even found to be considerably larger than for the ones with two symmetric tails. Furthermore, the DiDs with the shortest tails (three carbons and less) were oriented opposite to the longer ones - they faced the water phase with their nitrogen atoms and were in closer contact with water. Consequently, the absorption spectra calculated through the use of hybrid quantum mechanics - molecular mechanics approach showed a blue-shift for the short-tail DiD 1-1. Based on their macroscopic properties namely position and orientation, we showed here that the presented probes can be successfully used for optical microscopy in biological environments. Since also the phase preference of the probes might be affected by the lengths of the asymmetric tails, further systematic investigation into the consequences of the lengths of the alkyl chains is needed.

\section{Conflicts of interest}


There are no conflicts of interest to declare.

\section{Acknowledgments}

M. P. gratefully acknowledges the Special Research Fund (BOF) of Hasselt University (Belgium), where she stayed in November 2018. She thanks the Department of Theoretical Chemistry and Biology at KTH for her post doc fellowship. The computational time was provided by the Swedish National Infrastructure for Computing (SNIC) through the medium allocations 3-397, 3-156, 3-396, and 3-23 in 2018. The Flemish Supercomputer Centre (VSC) along with the Herculesstichting (Flanders, Belgium) provided thereupon access to the Breniac cluster. The authors thank Lindsay Leach for language consultation.

\section{Supporting Information}

Table S1: Extended Table 1 with average data from the simulations; Figure S1: RESP partial charges; Figure S2: Potential energy scans along three dihedral angles; Table S2: Ryckaert-Bellemans potential parameters; Figure S3: Evolution of DiD distance from the membrane center; Figure S4: Evolution of the orientation of DiD in respect of the z-axis; Figure S5: Orientation of DiD alkyl tails; Figure S6: Deuterium order parameters; Figure S7: Density distribution of DiD; Figure S8: DOPC density profile; Figure S9: Radial distribution function of water around DiD nitrogens; Figure S10: Projection of DiD transition dipole moment on the membrane plane; Figure S11: Additional simulations - Evolution of DiD distance from the membrane center; Figure S12: Additional simulations - Evolution of the orientation of DiD in respect of the z-axis; Figure S13: Standard deviations of positions of center of mass; Fluorescence anisotropy discussion; Topologies for all used DiD molecules.

\section{References}

[1] B. Alberts, A. Johnson, J. Lewis, M. Raff, K. Roberts, P. Walter, Molecular Biology of THE CELL, 5th Ed., New York: Garland Science, 2008.

[2] F. Di Meo, G. Fabre, K. Berka, T. Ossman, B. Chantemargue, M. Paloncýová, P. Marquet, M. Otyepka, P. Trouillas, In Silico Pharmacology: Drug Membrane Partitioning and Crossing, Pharmacol. Res., 111 (2016) 471-486 DOI: 10.1016/j.phrs.2016.06.030.

[3] P. Anzenbacher, E. Anzenbacherová, Cytochromes P450 and Metabolism of Xenobiotics, Cell. Mol. Life Sci., 58 (2001) 737-747 DOI: 10.1007/PL00000897.

[4] P.L. Yeagle, Membrane Proteins, in: Membr. Cells, Elsevier, 2016: pp. 219-268.

[5] G. van Meer, D.R. Voelker, G.W. Feigenson, Membrane Lipids : Where They Are and How They Behave, Nat. Rev. Mol. Cell Biol., 9 (2008) 112-124 DOI: 10.1038/nrm2330.

[6] H.-J. Kaiser, D. Lingwood, I. Levental, J.L. Sampaio, L. Kalvodova, L. Rajendran, K. Simons, Order of lipid phases in model and plasma membranes, Proc. Natl. Acad. Sci., 106 (2009) 16645-16650 DOI: 10.1073/pnas.0908987106.

[7] L.J. Pike, The Challenge of Lipid Rafts, J. Lipid Res., 50 Suppl (2009) S323-328 DOI: 10.1194/jlr.R800040-JLR200.

[8] S.N. Baker, G.A. Baker, Luminescent Carbon Nanodots : Emergent Nanolights, Angew. Chemie - Int. Ed., 49 (2010) 6726-6744 DOI: 10.1002/anie.200906623.

[9] A.S. Klymchenko, R. Kreder, Fluorescent probes for lipid rafts: From model membranes to living cells, Chem. Biol., 21 (2014) 97-113 DOI: 10.1016/j.chembiol.2013.11.009. 
[10] J.R. Lakowicz, Principles of fluorescence spectroscopy, 2006.

[11] J. Juhasz, J.H. Davis, F.J. Sharom, Fluorescent probe partitioning in GUVs of binary phospholipid mixtures: Implications for interpreting phase behavior, Biochim. Biophys. Acta Biomembr., 1818 (2012) 19-26 DOI: 10.1016/j.bbamem.2011.09.006.

[12] T. Baumgart, G. Hunt, E.R. Farkas, W.W. Webb, G.W. Feigenson, Fluorescence probe partitioning between Lo/Ld phases in lipid membranes, Biochim. Biophys. Acta - Biomembr., 1768 (2007) 2182-2194 DOI: 10.1016/j.bbamem.2007.05.012.

[13] N. Kahya, D. Scherfeld, K. Bacia, B. Poolman, P. Schwille, Probing Lipid Mobility of Raftexhibiting Model Membranes by Fluorescence Correlation Spectroscopy *, 278 (2003) 2810928115 DOI: 10.1074/jbc.M302969200.

[14] S. Knippenberg, G. Fabre, S. Osella, F. Di Meo, M. Paloncýová, M. Ameloot, P. Trouillas, Atomistic Picture of Fluorescent Probes with Hydrocarbon Tails in Lipid Bilayer Membranes: An Investigation of Selective Affinities and Fluorescent Anisotropies in Different Environmental Phases, Langmuir, 34 (2018) 9072-9084 DOI: 10.1021/acs.langmuir.8b01164.

[15] D. Axelrod, Carbocyanine dye orientation in red cell membrane studied by microscopic fluorescence polarization, Biophys. J., 26 (1979) 557-573 DOI: 10.1016/S00063495(79)85271-6.

[16] S. Osella, F. Di Meo, N.A. Murugan, G. Fabre, M. Ameloot, P. Trouillas, S. Knippenberg, Combining (Non)linear Optical and Fluorescence Analysis of DiD To Enhance Lipid Phase Recognition, J. Chem. Theory Comput., 14 (2018) 5350-5359 DOI: 10.1021/acs.jctc.8b00553.

[17] D.G. Ackerman, F.A. Heberle, G.W. Feigenson, Limited perturbation of a DPPC bilayer by fluorescent lipid probes: A molecular dynamics study, J. Phys. Chem. B, 117 (2013) 48444852 DOI: $10.1021 / \mathrm{jp} 400289 \mathrm{~d}$.

[18] R.R. Gullapalli, M.C. Demirel, P.J. Butler, Molecular dynamics simulations of DiI-C18(3) in a DPPC lipid bilayer, Phys. Chem. Chem. Phys., 10 (2008) 3548 DOI: 10.1039/b716979e.

[19] R. Faller, Molecular modeling of lipid probes and their influence on the membrane, Biochim. Biophys. Acta - Biomembr., 1858 (2016) 2353-2361 DOI: 10.1016/j.bbamem.2016.02.014.

[20] H.A.L. Filipe, L.S. Santos, J.P. Prates Ramalho, M.J. Moreno, L.M.S. Loura, Behaviour of NBD-head group labelled phosphatidylethanolamines in POPC bilayers: a molecular dynamics study, Phys. Chem. Chem. Phys., 17 (2015) 20066-20079 DOI: 10.1039/c5cp01596k.

[21] A. Kyrychenko, M. V. Rodnin, A.S. Ladokhin, Calibration of Distribution Analysis of the Depth of Membrane Penetration Using Simulations and Depth-Dependent Fluorescence Quenching, J. Membr. Biol., 248 (2015) 583-594 DOI: 10.1007/s00232-014-9709-1.

[22] H.A.L. Filipe, M.J. Moreno, L.M.S. Loura, Interaction of 7-nitrobenz-2-oxa-1,3-diazol-4-yllabeled fatty amines with 1-palmitoyl, 2-oleoyl-sn-glycero-3-phosphocholine bilayers: A molecular dynamics study, J. Phys. Chem. B, 115 (2011) 10109-10119 DOI: $10.1021 / \mathrm{jp} 203532 \mathrm{c}$.

[23] A. Kyrychenko, A molecular dynamics model of rhodamine-labeled phospholipid incorporated into a lipid bilayer, Chem. Phys. Lett., 485 (2010) 95-99 DOI: 10.1016/j.cplett.2009.12.015.

[24] S. Knippenberg, R.L. Gieseking, D.R. Rehn, S. Mukhopadhyay, A. Dreuw, J.-L. Brédas, Benchmarking Post-Hartree-Fock Methods To Describe the Nonlinear Optical Properties of Polymethines: An Investigation of the Accuracy of Algebraic Diagrammatic Construction (ADC) Approaches, J. Chem. Theory Comput., 12 (2016) 5465-5476 DOI: 10.1021/acs.jctc.6b00615.

[25] F. Meyers, S.R. Marder, B.M. Pierce, J.L. Bredas, Electric Field Modulated Nonlinear Optical 
Properties of Donor-Acceptor Polyenes: Sum-Over-States Investigation of the Relationship between Molecular Polarizabilities (.alpha, .beta, and .gamma) and Bond Length Alternation, J. Am. Chem. Soc., 116 (1994) 10703-10714 DOI: 10.1021/ja00102a040.

[26] B. Le Guennic, D. Jacquemin, Taking Up the Cyanine Challenge with Quantum Tools, Acc. Chem. Res., 48 (2015) 530-537 DOI: 10.1021/ar500447q.

[27] R. Send, O. Valsson, C. Filippi, Electronic Excitations of Simple Cyanine Dyes: Reconciling Density Functional and Wave Function Methods, J. Chem. Theory Comput., 7 (2011) 444-455 DOI: $10.1021 / \mathrm{ct} 1006295$.

[28] R.L. Gieseking, S. Mukhopadhyay, C. Risko, S.R. Marder, J.-L. Brédas, 25th Anniversary Article: Design of Polymethine Dyes for All-Optical Switching Applications: Guidance from Theoretical and Computational Studies, Adv. Mater., 26 (2014) 68-84 DOI: 10.1002/adma.201302676.

[29] S. Mukhopadhyay, C. Risko, S.R. Marder, J.L. Brédas, Polymethine dyes for all-optical switching applications: A quantum-chemical characterization of counter-ion and aggregation effects on the third-order nonlinear optical response, Chem. Sci., 3 (2012) 3103-3112 DOI: $10.1039 / \mathrm{c} 2 \mathrm{sc} 20861 \mathrm{j}$.

[30] S. Osella, S. Knippenberg, Triggering On/O ff States of Photoswitchable Probes in Biological Environments, J. Am. Chem. Soc., 139 (2017) 4418-4428 DOI: 10.1021/jacs.6b13024.

[31] J. Fu, L.A. Padilha, D.J. Hagan, E.W. Van Stryland, O. V Przhonska, M. V Bondar, Y.L. Slominsky, A.D. Kachkovski, Molecular structure - two-photon absorption property relations in polymethine dyes, J. Opt. Soc. Am. B, 24 (2007) 56 DOI: 10.1364/JOSAB.24.000056.

[32] R.W. Sabnis, Handbook of Biological Dyes and Stains: Synthesisand Industrial Applications, 2010 .

[33] L. Shan, Near-infrared fluorescence 1,1-dioctadecyl-3,3,3,3-tetramethylindotricarbocyanine iodide (DiR)-labeled macrophages for cell imaging, in: Mol. Imaging Contrast Agent Database, 2004: pp. 1-5.

[34] J. Wang, P. Cieplak, P.A. Kollman, How well does a restrained electrostatic potential (RESP) model perform in calculating conformational energies of organic and biological molecules?, J. Comput. Chem., 21 (2000) 1049-1074 DOI: 10.1002/1096-987X(200009)21:12<1049::AIDJCC3>3.0.CO;2-F.

[35] M.J. Frisch, G.W. Trucks, H.B. Schlegel, G.E. Scuseria, M.A. Robb, J.R. Cheeseman, G. Scalmani, V. Barone, G.A. Petersson, H. Nakatsuji, X. Li, M. Caricato, A. V. Marenich, J. Bloino, B.G. Janesko, R. Gomperts, B. Mennucci, D.J. Hratch, Gaussian 16, Revision B01, (2016).

[36] A.D. Becke, Density-Functional Exchange-Energy Approximation with Correct Asymptotic Behavior, Phys. Rev. A, 38 (1988) 3098-3100.

[37] T.H. Dunning, Gaussian basis sets for use in correlated molecular calculations I The atoms boron through neon and hydrogen, J. Chem. Phys., 90 (1989) 1007-1023 DOI: $10.1063 / 1.456153$.

[38] Y. Duan, C. Wu, S. Chowdhury, M.C. Lee, G. Xiong, W. Zhang, R. Yang, P. Cieplak, R. Luo, T. Lee, J. Caldwell, J. Wang, P. Kollman, A point-charge force field for molecular mechanics simulations of proteins based on condensed-phase quantum mechanical calculations, J. Comput. Chem., 24 (2003) 1999-2012 DOI: 10.1002/jcc.10349.

[39] D.A. Case, T.A. Darden, 3rd. Cheatham, T. E., C.L. Simmerling, J. Wang, R.E. Duke, R. Luo, R.C. Walker, W. Zhang, K.M. Merz, B. Roberts, B. Wang, S. Hayik, A. Roitberg, G. Seabra, I. Kolossváry, K.F. Wong, F. Paesani, J. Vanicek, J. Liu, et al., AMBER 11, University of 
California, San Francisco, (2010).

[40] M. Paloncýová, G. Fabre, R.H. Devane, P. Trouillas, K. Berka, M. Otyepka, Benchmarking of Force Fields for Molecule - Membrane Interactions, J. Chem. Theory Comput., 10 (2014) 4143-4151 DOI: $10.1021 / \mathrm{ct} 500419 b$ |.

[41] S.-W. Chiu, S.A. Pandit, H.L. Scott, E. Jakobsson, An Improved United Atom Force Field for Simulation of Mixed Lipid Bilayers, J. Phys. Chem. B, 113 (2009) 2748-2763 DOI: 10.1021/jp807056c.

[42] M. Paloncýová, M. Ameloot, S. Knippenberg, Orientational distribution of DPH in lipid membranes: a comparison of molecular dynamics calculations and experimental time-resolved anisotropy experiments, Phys. Chem. Chem. Phys., 21 (2019) 7594-7604 DOI: 10.1039/C8CP07754A.

[43] S.A. Pandit, S.-W. Chiu, E. Jakobsson, A. Grama, H.L. Scott, Cholesterol Packing around Lipids with Saturated and Unsaturated Chains: a Simulation Study, Langmuir, 24 (2008) 6858-6865 DOI: 10.1021/la8004135.

[44] S.A. Pandit, S.-W. Chiu, E. Jakobsson, A. Grama, H.L. Scott, Cholesterol Surrogates: a Comparison of Cholesterol and 16:0 Ceramide in POPC Bilayers, Biophys. J., 92 (2007) 920927 DOI: 10.1529/biophysj.106.095034.

[45] S.A. Pandit, E. Jakobsson, H.L. Scott, Simulation of the Early Stages of Nano-domain Formation in Mixed Bilayers of Sphingomyelin, Cholesterol, and Dioleylphosphatidylcholine, Biophys. J., 87 (2004) 3312-3322 DOI: 10.1529/biophysj.104.046078.

[46] H.J.C. Berendsen, J.R. Grigera, T.P. Straatsma, The Missing Term in Effective Pair Potentials, J. Phys. Chem., 91 (1987) 6269-6271 DOI: 10.1021/j100308a038.

[47] D. Van Der Spoel, E. Lindahl, B. Hess, G. Groenhof, A.E. Mark, H.J.C. Berendsen, GROMACS: Fast, Flexible, and Free, J. Comput. Chem., 26 (2005) 1701-1718 DOI: 10.1002/jcc.20291.

[48] S. Nosé, A Unified Formulation of the Constant Temperature Molecular Dynamics Methods, J. Chem. Phys., 81 (1984) 511-519 DOI: 10.1063/1.447334.

[49] W.G. Hoover, Canonical Dynamics: Equilibrium Phase-Space Distributions, Phys. Rev. A, 31 (1985) 1695-1697.

[50] M. Parrinello, A. Rahman, Polymorphic Transitions in Single Crystals: A New Molecular Dynamics Method, J. Appl. Phys., 52 (1981) 7182-7190 DOI: 10.1063/1.328693.

[51] T. Darden, D. York, L. Pedersen, Particle Mesh Ewald: An N.log(N) Method for Ewald Sums in Large Systems, J. Chem. Phys., 98 (1993) 10089-10092 DOI: 10.1063/1.464397.

[52] B. Hess, H. Bekker, H.J.C. Berendsen, J.G.E.M. Fraaije, LINCS: A Linear Constraint Solver for Molecular Simulations, J. Comput. Chem., 18 (1997) 1463-1472 DOI: 10.1002/(SICI)1096-987X(199709)18:12<1463::AID-JCC4>3.3.CO;2-L.

[53] T. Yanai, D.P. Tew, N.C. Handy, A new hybrid exchange-correlation functional using the Coulomb-attenuating method (CAM-B3LYP), Chem. Phys. Lett., 393 (2004) 51-57 DOI: 10.1016/j.cplett.2004.06.011.

[54] S. Osella, N.A. Murugan, N.K. Jena, S. Knippenberg, Investigation into Biological Environments through (Non)linear Optics: A Multiscale Study of Laurdan Derivatives, J. Chem. Theory Comput., 12 (2016) 6169-6181 DOI: 10.1021/acs.jctc.6b00906.

[55] N.A. Murugan, R. Apostolov, Z. Rinkevicius, J. Kongsted, E. Lindahl, H. Ågren, Association dynamics and linear and nonlinear optical properties of an $\mathrm{N}$-acetylaladanamide probe in a POPC membrane, J. Am. Chem. Soc., 135 (2013) 13590-13597 DOI: 10.1021/ja407326n. 
[56] K. Aidas, C. Angeli, K.L. Bak, V. Bakken, R. Bast, L. Boman, O. Christiansen, R. Cimiraglia, S. Coriani, P. Dahle, E.K. Dalskov, U. Ekström, T. Enevoldsen, J.J. Eriksen, P. Ettenhuber, B. Fernández, L. Ferrighi, H. Fliegl, L. Frediani, K. Hald, et al., The Dalton quantum chemistry program system, Wiley Interdiscip. Rev. Comput. Mol. Sci., 4 (2014) 269-284 DOI: 10.1002/wcms.1172.

[57] J.M.H. Olsen, J. Kongsted, Molecular Properties through Polarizable Embedding, 2011.

[58] J.P. Ryckaert, A. Bellemans, Molecular dynamics of liquid n-butane near its boiling point, Chem. Phys. Lett., 30 (1975) 123-125 DOI: 10.1016/0009-2614(75)85513-8.

[59] T.J. Piggot, J.R. Allison, R.B. Sessions, J.W. Essex, On the Calculation of Acyl Chain Order Parameters from Lipid Simulations, J. Chem. Theory Comput., 13 (2017) 5683-5696 DOI: 10.1021/acs.jctc.7b00643.

[60] S.-J. Marrink, H.J.C. Berendsen, Simulation of Water Transport through a Lipid Membrane, J. Phys. Chem., 98 (1994) 4155-4168 DOI: 10.1021/j100066a040.

[61] J. Rodríguez, D. Scherlis, D. Estrin, P.F. Aramendía, R.M. Negri, AM1 study of the ground and excited state potential energy surfaces of symmetric carbocyanines, J. Phys. Chem. A, 101 (1997) 6998-7006 DOI: 10.1021/jp9713569.

\section{Highlights}

- MD on dyes with cyanine backbone, asymmetric tails, increasing length of bridge

- Hybrid QM/MM calculations to investigate influence of environment on spectrum

- Probes with both tails shorter or equal to 3 carbons are oriented towards water

- Photoselection with incoming field parallel to surface most effective for DiD 6-3

- Blue shift of $40 \mathrm{~nm}$ in absorption seen due to particular orientation of DiD 1-1

TOC

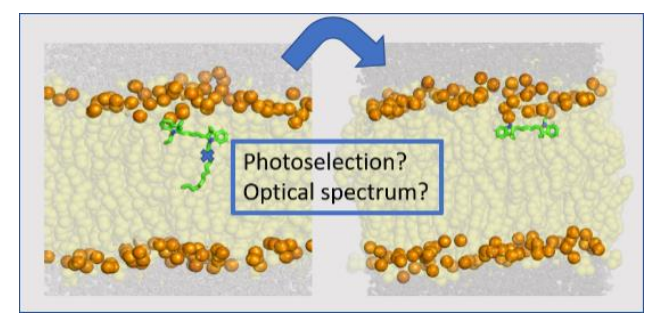

